\section{Contribution of $B C R-A B L$ kinase domain mutations to imatinib mesylate resistance in Philadelphia chromosome positive Malaysian chronic myeloid leukemia patients}

\author{
Marjanu Hikmah Elias, ${ }^{1}$ \\ Abdul Aziz Baba, ${ }^{2}$ Azlan Husin, ${ }^{2}$ \\ Abu Dzarr Abdullah, ${ }^{2}$ Rosline Hassan, ${ }^{3}$ \\ Goh Ai Sim, ${ }^{4}$ S. Fadilah Abdul Wahid, ${ }^{5}$ \\ Ravindran Ankathil ${ }^{1}$ \\ ${ }^{1}$ Human Genome Centre; ${ }^{2}$ Haemato- \\ Oncology Unit, Department of Internal \\ Medicine; ${ }^{3}$ Hematology Department, \\ School of Medical Sciences, Health \\ Campus, Universiti Sains Malaysia; \\ ${ }^{4}$ Hospital Pulau Pinang; ${ }^{5}$ Medicine \\ Department and Cell Therapy Centre, \\ UKM Medical Centre, Malaysia
}

\section{Abstract}

Development of resistance to imatinib mesylate (IM) in chronic myeloid leukemia (CML) patients is mediated by different mechanisms that can be classified as BCR-ABL dependent or $B C R-A B L$ independent pathways. $B C R-A B L$ dependent mechanisms are most frequently associated with point mutations in tyrosine kinase domain (TKD) of BCR-ABL1 and also with $B C R-A B L$ gene amplification. Many different types and frequencies of mutations have been reported in different studies, probably due to the different composition of study cohorts. Since no reports are available from Malaysia, this study was undertaken to investigate the frequency and pattern of $B C R$ $A B L$ kinase domain mutations using dHPLC followed by sequencing, and also status of $B C R-A B L$ gene amplification using fluorescence in situ hybridization (FISH) on 40 IM resistant Malaysian CML patients. Mutations were detected in 13 patients (32.5\%). Five different types of mutations (T315I, E255K, $\mathrm{Y} 253 \mathrm{H}, \mathrm{M} 351 \mathrm{~T}, \mathrm{~V} 289 \mathrm{~F}$ ) were identified in these patients. In the remaining $27 \mathrm{IM}$ resistant CML patients, we investigated the contribution made by $B C R-A B L$ gene amplification, but none of these patients showed amplification. It is presumed that the mechanisms of resistance in these 27 patients might be due to $B C R$ $A B L$ independent pathways. Different mutations confer different levels of resistance and, therefore, detection and characterization of TKD mutations is highly important in order to guide therapy in CML patients.

\section{Introduction}

Imatinib mesylate (IM) is used as a frontline therapy for chronic myeloid leukemia (CML) as it is highly effective in the treatment and management of Philadelphia ( $\mathrm{Ph}$ ) positive CML patients. IM is a selective inhibitor of tyrosine kinase that binds competitively to the adenosine triphosphate (ATP) docking site of tyrosine kinase proteins, including ABL itself and the hybrid BCR-ABL proteins. It also inhibits other tyrosine kinases, such as platelet-derived growth factor receptors KIT and ARG. ${ }^{1}$ As IM binds to the ATP binding site at the tyrosine kinase active site, it inhibits the transfer of terminal phosphate from ATP to tyrosine residues on its substrates, thus inhibiting the enzyme activity of the tyrosine kinase protein semi-competitively. Mutations in the $B C R-A B L$ kinase domain can cause resistance to IM by shifting its equilibrium toward the open or active conformation. Some other mutations interfere with a critical hydrogen bond that forms between the $A B L 1$ kinase domain and inhibitor molecule thus impairing the ability of the BCR-ABL protein to bind to the inhibitor molecule. ${ }^{2}$

Even though IM has become the gold standard in front-line treatment of CML, resistance to this drug is a daunting problem. Development of resistance to IM has been a setback for patients, as well as for treating physicians in the clinical management of CML patients. A patient with CML could display either a primary or secondary resistance to IM therapy. Primary resistance is defined as inability to achieve any initial landmark response, and secondary response is defined as achieving a good initial response but subsequently losing any relevant response. ${ }^{3}$

Development of resistance to IM is a multifactorial phenomenon in patients with CML and may be mediated by a range of different mechanisms. However, there are 2 broad mechanisms of resistance: $B C R-A B L$ dependent and $B C R-A B L$ independent pathways.,5 $B C R-A B L$ dependent pathways have been reported to be the most common cause of IM resistance which commonly involve mutations in the tyrosine kinase domain (TKD) of the $B C R-A B L$ gene, ${ }^{6}$ as well as amplification and overexpression of the $B C R-A B L$ gene locus. ${ }^{7}$ The present study was designed to clarify the mechanisms of resistance involving $B C R-A B L$ dependent pathways.

So far, more than 100 mutations have been identified in the tyrosine kinase domain of the $B C R-A B L$ gene. ${ }^{8}$ Different studies have reported a broad range of frequencies of mutations and this is probably due to the different composition of study cohorts. But no reports are available from Malaysia. Apart from BCR-ABL
Correspondence: Ravindran Ankathil, Human Genome Centre, School of Medical Sciences, Health Campus, Universiti Sains Malaysia, 16150 Kubang Kerian, Kelantan, Malaysia.

Tel. +609.7676968 - Fax: +609.7658914.

E-mail: rankathil@hotmail.com

Contributions: MHE collected samples, performed research and drafted the paper; $\mathrm{AAB}, \mathrm{AH}, \mathrm{ADA}$, $\mathrm{RH}, \mathrm{GAS}$ and SFAW helped in patient recruitment, confirmed clinical status and interpreted clinical data; RA designed the research, and corrected and revised the paper.

Key words: chronic myeloid leukemia, imatinib mesylate, BCR-ABL dependent mechanisms, tyrosine kinase domain, mutation.

Acknowledgments: this study was supported by the Ministry of Higher Education (MOHE), Malaysia, Fundamental Research Grant Scheme (FRGS) 203/PPSP/6171104. The first author is a Universiti Sains Malaysia Fellowship holder. The contributions of Human Genome Center staff, Universiti Sains Malaysia and all the patients who have participated in this study are gratefully acknowledged.

Conflict of interests: the authors report no potential conflict of interests.

Received for publication: 23 July 2012.

Revision received: 23 October 2012.

Accepted for publication: 31 October 2012.

This work is licensed under a Creative Commons Attribution NonCommercial 3.0 License (CC BYNC 3.0).

(C) Copyright M.H. Elias et al., 2012

Licensee PAGEPress, Italy

Hematology Reports 2012; 4:e23

doi:10.4081/hr.2012.e23

mutation, few studies have reported on the amplification of the BCR-ABL gene locus that is associated with IM resistance among CML patients. ${ }^{9}$ In these rare cases, the presence of multiple copies of the $B C R-A B L$ gene in interphase nuclei were reported in IM resistant patients using fluorescence in situ hybridization (FISH). In this study, we investigated the frequency and pattern of $B C R-A B L$ kinase domain mutations using $\mathrm{dHPLC}$ and $B C R-A B L$ gene amplification by FISH on 40 Malaysian CML patients who showed resistance to IM.

\section{Materials and Methods}

\section{Study subjects}

The study was undertaken at Universiti Sains Malaysia Hospital from 2008 to 2011, after obtaining approval from the institute's Research and Ethics Committee. The study 
participants included 40 Philadelphia chromosome $(\mathrm{Ph})$ positive CML patients in chronic, accelerated or blast phase, treated for at least six months with standard dose IM (400 mg) as front-line treatment according to the phase II extended access protocols, and who showed only suboptimal response or signs of clinical response to IM. Those CML patients who were $\mathrm{Ph}$ negative, and those who did not opt for IM treatment, were excluded from the study. The medical records of all patients were reviewed until June 2011. Basic demographic, disease characteristics, and treatment management details were collected. For each patient, diagnosis was confirmed by hematologic, cytogenetic, as well as molecular analysis. The response to IM therapy was evaluated on the basis of measurement of hematologic, cytogenetic and molecular responses. Hematologic response was evaluated every $3^{\text {rd }}$ month of treatment and cytogenetic response was evaluated every $6^{\text {th }}$ month of treatment.

According to European LeukemiaNet 2010, complete hematologic remission would show peripheral blood cell counts and bone marrow morphology returning to normal with total white blood cell count less than $10 \times 10^{9} / \mathrm{L}$ and platelet count less than $450 \times 10^{9} / \mathrm{L}$. Complete hematologic remission was also defined by absence of peripheral blast, immature granulocytes such as promyelocytes or myelocytes, less than 5\% peripheral basophils and non-palpable spleen. ${ }^{10}$ Cytogenetic remission was categorized into complete, major, partial, minor and non-responder groups. A total disappearance of $\mathrm{Ph}$ chromosome in cytogenetic analysis confirmed complete cytogenetic response (CCyR) while presence of less than $35 \% \mathrm{Ph}^{+}$cells in bone marrow confirmed partial cytogenetic response (PCyR). Patients with minor cytogenetic response showed $36-65 \%$ of $\mathrm{Ph}^{+}$cells in bone marrow while those who showed $66-95 \%$ $\mathrm{Ph}^{+}$chromosome positivity were categorized as a minimal cytogenetic response group. Patients whose bone marrow showed over $95 \%$ $\mathrm{Ph}^{+}$chromosome were classified as nonresponders to IM. $^{10}$

Primary resistance, also known as intrinsic resistance to IM, is defined as having no hematologic response within three months, having incomplete hematologic response or no cytogenetic response within six months, and having less than partial cytogenetic response within 12 months. Secondary resistance or acquired resistance is defined as evolution of the disease from chronic phase to accelerated phase or blast phase, loss of hematologic response, loss of MCyR or CCyR. ${ }^{11}$

\section{$B C R-A B L$ mutation analysis}

$B C R-A B L$ mutation analysis was carried out in both CML patients showing primary and secondary resistance using the denaturing high performance liquid chromatography (dHPLC) method. For this, total RNA was extracted from the peripheral blood using the QIAamp RNA blood mini extraction kit (QIAGEN, Germany) according to the manufacturer's instructions with a slight modification, followed by cDNA synthesis using the cDNA Synthesis kit (Bioline, UK) with a slightly modified protocol. Subsequently, amplification of 3 overlapping fragments covering the entire kinase domain was generated by nested PCR using the primers described by Soverini et. $a l .{ }^{12}$ The first PCR (BCR-A) with 1475 bp amplicon length was amplified from the synthesized cDNA, followed by the second PCR (ABL-B) with 393 bp amplicon length, amplified from BCR-A amplicon. The third PCR (ABL-C) with 482 bp amplicon length was subsequently amplified, also from BCR-A amplicon. All PCR procedures were performed using AccuSure Mix (Bioline, UK).

The presence of sequence variation was then screened by dHPLC in which PCR product was mixed with wild-type sample (Ph positive sample with no mutation identified after sequencing) and denatured to allow the formation of heteroduplex. This was then analyzed under the optimum melting temperature that was determined using a melting program (http://insertion.stanford.edu/melt.htm). Subsequently, samples were screened by dHPLC (ProStar Helix System, Varian, USA). The PCR products of samples that showed altered dHPLC profile, indicative of mutation, were directly sequenced with both forward and reverse primers after purification steps using a PCR purification kit (QIAGEN, Germany) to characterize the mutation. Samples with mutation were then resequenced for confirmation.

\section{$B C R-A B L$ gene amplification}

The samples of patients who did not show any $B C R-A B L$ mutations were investigated for the progressive amplification status of the $B C R-A B L$ gene using FISH. For this, LSI BCR$A B L$ dual fluorescent probes (Vysis Inc., USA) which hybridize to the fusion regions and generate intense fluorescence signals were used. The copy number of fusion signals in a minimum of 200 interphase nuclei was determined for each sample.

\section{Bioinformatics analysis}

An online program (available at http://genetics.bwh.harvard.edu/pph2/), PolyPhen-2, was used to predict the potential consequence of each mutation on the BCR-ABL protein structure. ClustalX program (version 2.0.12) was used for multiple alignment of Homo sapiens ABL1 protein sequence (CAA34438) with ABL1 protein sequence of chimpanzee (Pan troglodytes; XP_001166213.2), pig (Sus scrofa; XP_003122293.3), mouse (Mus musculus;
NP_001106174.1), rat (Rattus norvegicus; NP_001094320.1), cow (Bos taurus; NP_001193789.1) and chicken (Gallus gallus; XP_001233812.1).

\section{Results}

The demographic profile, disease characteristics, treatment management, cytogenetic analysis and molecular analysis of all patients participating in the study are shown in Tables 1 and 2. The ratio of male to female CML

Table 1. Demographic, disease characteristics and treatment management of the chronic myeloid leukemia patients.

\begin{tabular}{lc} 
Demographic & N. patients (\%) \\
Gender & \\
Male & $16(40)$ \\
Female & $24(60)$ \\
Age & \\
Median (range) & $43(17-65)$ \\
Male & $41(17-65)$ \\
Female & $45(23-65)$ \\
\hline Race & \\
Malay & $34(85)$ \\
Chinese & $5(12.5)$ \\
Indian & $1(2.5)$ \\
Treatment duration (month) & \\
$13-24$ & $1(2.5)$ \\
$25-36$ & $3(7.5)$ \\
$37-48$ & $6(15)$ \\
$49-60$ & $14(35)$ \\
$>61$ & $16(40)$ \\
\hline Disease characteristic and & \\
treatment management &
\end{tabular}

CML stage prior to IM

Chronic phase

Accelerated phase

$37(92.5)$

Blast phase

$3(7.5)$

\begin{tabular}{lc} 
Treatment prior to IM & \\
IFN & $2(5.0)$ \\
Hydroxyurea & $21(52.5)$ \\
No & $17(42.5)$ \\
\hline
\end{tabular}

CML stage in response to IM

Chronic phase

Accelerated phase $\quad 6(15)$

Blast phase 3 (7.5)

Hematologic response (HR)

Complete 34 (85)

Suboptimal $\quad 5(12.5)$

Loss of HR 1 (2.5)

Cytogenetic response (CR)

$\begin{array}{lr}\text { No } & 10(25.0) \\ \text { Major/partial } & 4(10.0) \\ \text { Minor } & 11(27.5) \\ \text { Minimal } & 10(25.0) \\ \text { Complete but loss CyR } & 5(12.5) \\ \text { Resistance type } & \\ \text { Primary resistance } & 35(87.5) \\ \text { Secondary resistance } & 5(12.5)\end{array}$

CML, chronic myeloid leukemia; IM, imatinib mesylate. 
patients recruited was 2:3; mean age was 41 and 45 years, respectively. A slight predominance of the disease among females was observed.

In this cross-sectional study, out of 40 Philadelphia chromosome positive CML patients, mutations were detected in 13 (32.5\%). Among these 13, 8 (70\%) showed T315I mutation. Another 5 patients showed E255K ( $\mathrm{n}=2), \mathrm{Y} 253 \mathrm{H}, \mathrm{M} 351 \mathrm{~T}$ and V289F (1 each) mutations, respectively (Figure 1). The remaining 27 patients who did not show tyrosine kinase domain mutation were subjected to $B C R-A B L$ gene amplification using FISH. However, none of these 27 IM resistant patients showed $B C R-A B L$ gene amplification.

Cytogenetic analysis of the bone marrow samples was carried out in all these IM resistant CML patients of whom 13 showed additional chromosomal abnormalities apart from the Philadelphia chromosome. The additional chromosomal abnormalities included: trisomy 6 ( $\mathrm{n}=1$ patient), trisomy $8(\mathrm{n}=1)$, trisomy 10 $(\mathrm{n}=3)$, trisomy $16 \quad(\mathrm{n}=1)$ and trisomy 19 $(\mathrm{n}=2) ;$ monosomy $4(\mathrm{n}=2), \operatorname{del}(15)(\mathrm{q} 22-26)$ $(\mathrm{n}=1)$, del(17)(q23-25) $(\mathrm{n}=1)$ and $\mathrm{i}(17 \mathrm{q} 10)$ $(n=1)$. However, a comparison of the ACA (with or without ACA) with mutation (with or without mutation) showed no significant difference $(\mathrm{P}=0.646)$.

\section{Discussion}

Reciprocal translocation between chromosomes 9 and $22, \mathrm{t}(9: 22)(\mathrm{q} 34 ; \mathrm{q} 11)$ is reported to be the main factor contributing to CML. This unique chromosome arrangement, known as Philadelphia chromosome translocation, generates a $B C R-A B L$ fusion gene that encodes a constitutively active tyrosine kinase protein. $B C R-A B L$ fusion gene has now become the universally accepted molecular signature and the transforming event in CML pathogenesis. The mRNA molecules transcribed from $B C R$ $A B L$ fusion gene usually contain one of the two $B C R-A B L$ junctions designated as $\mathrm{b} 2 \mathrm{a} 2$ (e13a2) and b3a2 (e14a2). However, both mRNAs translate into p210 Kda fusion protein that may up-regulate the tyrosine kinase activity. This results in transformed cells that have growth factor independent proliferation along with decreased apoptosis, defective adhesion, as well as genomic instability.

In the present study, we aimed to examine the frequency distribution and nature of $A B L$ kinase domain mutation in CML patients treated with IM. The ability to detect mutations in the $B C R-A B L 1$ gene varies depending on the technique used. In the present study, we used the dHPLC technique for mutation analysis, followed by sequencing to characterize the mutations. The dHPLC assay provided a much
Table 2. Cytogenetic and molecular test results of the chronic myeloid leukemia patients.

\begin{tabular}{lc} 
Cytogenetic and molecular results & \\
Cytogenetics analysis (Bone marrow) & \\
t(9;22)(q34;q11) only & $27(67.5)$ \\
Additional chromosomal abnormalities & $13(32.5)$ \\
Mutation at $B C R-A B L$ gene & \\
Absent & $27(67.5)$ \\
Present & $13(32.5)$ \\
\hline Type of $B C R-A B L$ TKD & \\
T315I & $8(61.5)$ \\
E255K & $2(15.4)$ \\
M351T & $1(7.7)$ \\
Y253H & $1(7.7)$ \\
V289F & $1(7.7)$ \\
Occurrence of mutation & \\
Primary resistance & $12(92.3)$ \\
Secondary resistance & $1(7.7)$ \\
\hline BCR-ABL gene fusion transcript & \\
b3a2 & $31(77.5)$ \\
b2a2 & $7(17.5)$ \\
ela2 & $2(5.0)$ \\
\hline
\end{tabular}

a)

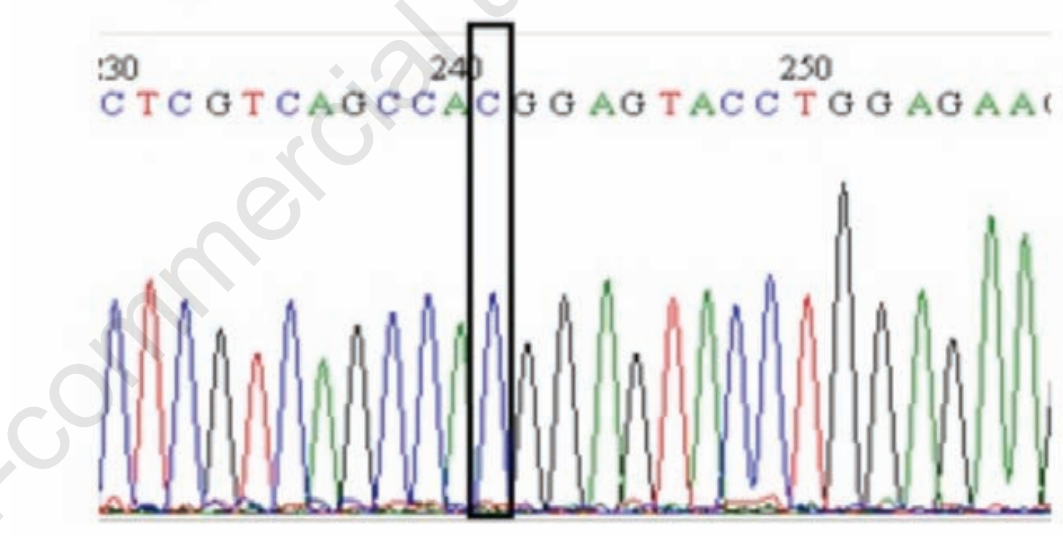

b)

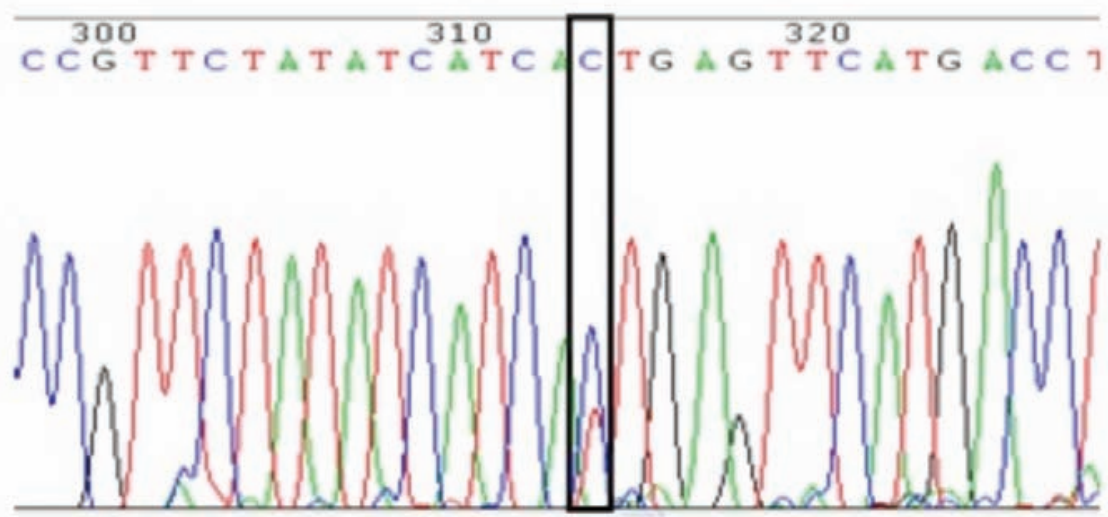

Figure 1. Sequencing analysis results of $B C R-A B L$ gene showing: a) M351T mutation; and b) T315I mutation. The circles indicate the nucleotide triplet that encodes the amino acid. 
faster and less expensive method for mutation screening. Taking advantage of the dHPLC based assay for $A B L$ mutational screening, as well as a sequencing technique, we screened 40 patients who showed resistance to IM. Our results showed that $\mathrm{ABL}$ kinase mutations are a relevant mechanism of resistance to IM in $32.5 \%$ of Malaysian CML patients.

Worldwide, researchers have detected quite a number of mutations within the $B C R-A B L$ fusion domain among IM resistant CML patients. In the present study on 40 Malaysian CML patients showing IM resistance, we detected a total of five different point mutations all leading to amino acid substitutions. Most of these mutations were found to be located at the functional fraction of the BCRABL fusion protein. Point mutations in $A B L$ kinase domain have been characterized into two groups: ${ }^{13}$ 1) mutations that impede contact between BCR-ABL and IM; and 2) mutations that alter the spatial conformation of the protein. ${ }^{7}$ The BCR-ABL structure contains two flexible loop structures, the adenosine triphosphate-binding phosphate loop and the activation loop, which have specific arrangement in the inactive conformation of BCR-ABL that stabilizes the structure. ${ }^{9}$ According to Litzow, IM mutations in these loops destabilize their arrangement such that the kinase domain cannot assume the inactive conformation required for IM binding. ${ }^{9}$

Mutations located in the IM contact point were reported to significantly reduce IM sensitivity. Normally, these amino acids make hydrogen bonds with IM; therefore, the mutations occurring in one of the amino acids might be causing resistance. ${ }^{2}$ This type of mutation was found in 8 of our patients who showed T315I mutation. From the literature reviewed, T315I was found to be the most common mutation reported worldwide and it also happened to be the most common mutation (70\%) among the IM resistant CML patients in the present study. ${ }^{14-16}$ In T315I mutation, a polar amino acid that participated in hydrogen bonds (threonine) is substituted with isoleucine, which is the hydrophobic amino acid. Thus, this single amino acid substitution has been reported to interfere with a critical hydrogen bond that forms between the $A B L 1$ kinase domain and IM. ${ }^{2}$ This allocated binding prevented the IM inhibition of BCR-ABL1 and conferred resistance to IM.

The detection of $B C R-A B L$ T315I mutation in the present study is clinically relevant. PolyPhen2 with a score of 0.999 using the HumDiv model (http://genetics.bwh.harvard. edu/pph2/) predicted the T315I mutation to be possibly damaging. Indeed, residue 315 seems to be critical for binding most adenosine triphosphate-competitive kinase inhibitors because $B C R-A B L$ T315I confers resistance to $2^{\text {nd }}$ generation tyrosine kinase inhibitors such as nilotinib and many other drugs. ${ }^{17}$

Other than T315I mutation, V289F mutation is also located at the IM binding site. In this mutation, valine, a very hydrophobic amino acid at position 289 is substituted with phenylalanine that is also a very hydrophobic amino acid. Like T315I, V289F was also predicted to be possibly damaging when analyzed using PolyPhen2 program with a score of 0.999 using the HumDiv model (http://genetics.bwh.harvard.edu/pph2/).

Another mutation is M351T. In this mutation at 351 , amino acid methionine, a very hydrophobic amino acid, is substituted with a less hydrophobic amino acid (threonine). Using the PolyPhen2 program, this type of mutation was predicted to be possibly damaging with a score of 1.00 using the HumDiv model. In a study on a Singaporean population, Ang et al. reported that M351T mutation was uncommon in Asian IM resistant CML patients, including Chinese, Malay, Indian and other Asian ethnics origins, as no M351T mutation was found in their study. ${ }^{18}$ However, interestingly, one of our patients (of Malay ethnic race) was found to have M351T mutation within the IM binding site of BCR-ABL kinase domain.

Mutations were also found in the P-loop of the BCR-ABL fusion domain. The residues of nucleotide binding loop (p-loop) were reported to be from 247 to $256 .{ }^{19}$ In our study, 2 patients showed different p-loop mutations which were Y253H and E255K mutations, respectively. Both of these mutations were predicted to be possibly damaging by using the PolyPhen2 program. Patients with mutated p-loop $B C R-A B L$ domain have been reported to be 70 to 100 -fold less sensitive to IM compared to native $B C R$ $A B L .{ }^{20}$ Other than that, mutations at the activation loop (a-loop) that include the residues from 381 until 402 in $A B L$ have also been reported to cause different levels of IM sensitivity among CML patients. ${ }^{21}$ However, none of our patients harbored this type of mutation.

Location of a mutation in a protein sequence may reflect the effect to the protein functions. Due to evolution, some proteins that play a similar biological function in different species may have significantly different protein sequences. However, part of the protein sequences in most species is preserved to retain the 3-dimensional structure, as well as the structure that is critical for conducting the biological function. Thus, these parts of protein sequences are usually conserved across species.22 The multi-alignment of human ABL1 protein with its orthologs among various species, using the ClustalX program (version 2.0.12), showed that all five mutations (T315I, M351T, E255K, Y253H and V289F) that we identified in our patients are conserved among species in a highly conserved block (Figure 2). From this, it is reasonable to presume that these mutations may lead to alteration of the BCR-ABL protein structure or important structure for its biological function that may affect the action of IM on this protein.

The RT-PCR amplification of the TKD domain of the $B C R-A B L$ gene in this study was performed by employing the primers described by Soverini et al. which covers only codon 206$421 .^{12}$ Even though most of $B C R-A B L$ mutations reported worldwide occurred in the TKD, mutations in the region of codon 421-500 which

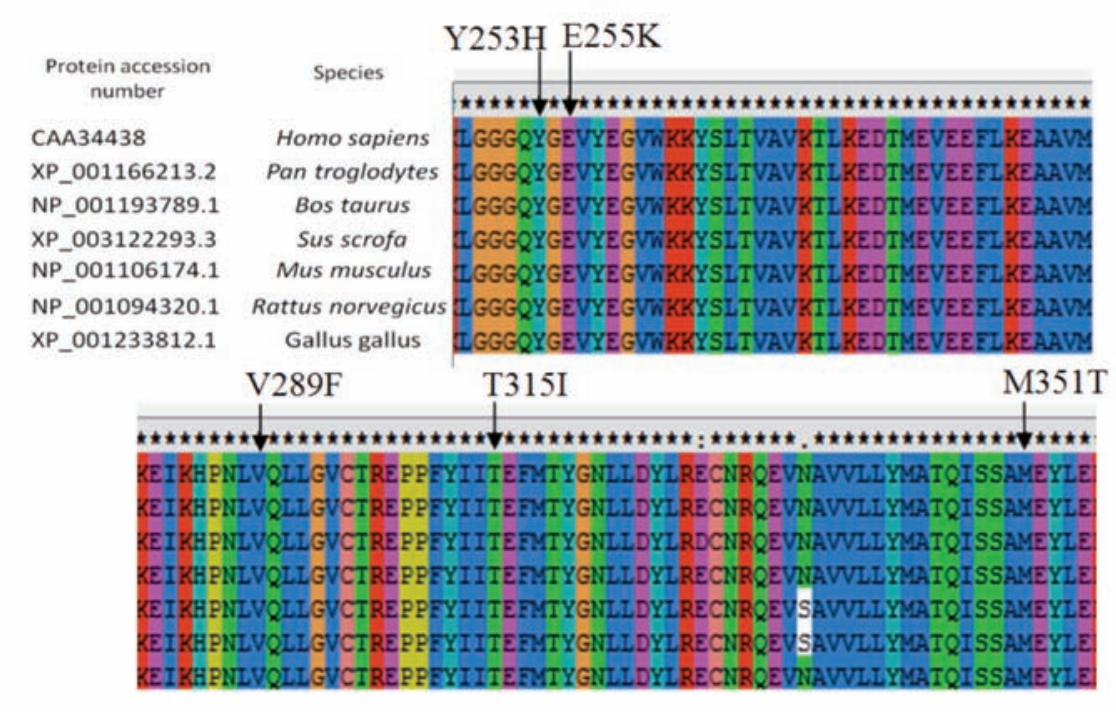

Figure 2. The multi-alignment of human ABL1 protein with its orthologs of various species by using ClustalX program (version 2.0.12). All the mutations detected are highly conserved among different species and located in a conserved block of amino acids. 
encode C-terminal lobe (i.e. E453K, Y456C and K459Q) have also been reported. ${ }^{23}$ Apart from the c-terminal, it has been reported that mutations could also occur at the $\mathrm{SH} 2-\mathrm{SH} 3$ domain (i.e. A196V, R47C and K84N). ${ }^{24}$ Unfortunately, in the current study, these 2 regions were not included in mutation screening. Had these 2 regions also been screened for mutations, it is probable that the mutation frequency would have been higher. Thus, for a better overview of the $B C R-A B L$ mutation, apart from the tyrosine kinase domain, both c-terminal and SH2-SH3 domain regions should also be taken into consideration. From these results, it is clear that the $B C R-A B L$ mutations contributed at least in part to resistance to IM in $32.5 \%$ of our patients. However, in those patients without kinase domain mutations, it is likely that additional factors may also contribute to resistance. In the remaining 27 patients who did not show any mutations, the contribution of $B C R-A B L$ gene amplification in mediating resistance was investigated using FISH. But none of these 27 patients showed any $B C R-A B L$ gene amplification. It is presumed that in these $27 \mathrm{CML}$ patients, the mechanism of resistance to IM might be due to $B C R$ - $A B L$ independent pathways. Another possibility could be the lower sensitivity of the FISH method. The FISH probes used are significantly larger than the BCR-ABL fusion region. Consequently, the gene amplification of BCR-ABL might not always be visible. Therefore, the usage of quantitative PCR performed on DNA is highly recommended to detect BCR-ABL gene amplification. Our results showed that BCR-ABL mutations are not the only major mechanisms of resistance to IM in Malaysian CML patients. Additional factors besides kinase domain mutations may also contribute to resistance to IM and this means we need to find out what other predominant mechanisms of IM resistance are involved.

\section{Conclusions}

Different mutations confer different levels of resistance and, therefore, detection as well as characterization of TKD mutations is highly important in order to guide therapy in CML patients. Knowing the exact mutations responsible for IM resistance will help to select the most suitable TKIs for CML patients and improve their management. Furthermore, early detection of such mutations may allow timely treatment intervention to prevent or overcome resistance.

\section{References}

1. Druker BJ, Guilhot F, 0'Brien SG, et al. Five-year follow-up of patients receiving imatinib for chronic myeloid leukemia. $\mathrm{N}$ Engl J Med 2006;355:2408-17.

2. Nagar B, Bornmann WG, Pellicena P, et al. Crystal structures of the kinase domain of c-Abl in complex with the small molecule inhibitors PD173955 and imatinib (STI571). Cancer Res 2002;62:4236-43.

3. Goldman JM. Chronic myeloid leukemia: a historical perspective. Semin Hematol 2010;47:302-11.

4. Hochhaus A. Chronic myelogenous leukemia (CML): resistance to tyrosine kinase inhibitors. Ann Oncol 2006;17: x274-9.

5. Mauro MJ. Defining and managing imatinib resistance. Hematol Am Soc Hematol Educ Program 2006:219-25.

6. La Rosee P, Deininger MW. Resistance to imatinib: mutations and beyond. Semin Hematol 2010;47:335-43.

7. Kantarjian HM, Talpaz M, Giles F,. New insights into the pathophysiology of chronic myeloid leukemia and imatinib resistance. Ann Intern Med 2006;145:913-23.

8. Mathisen MS, Kantarjian HM, Cortes J, Jabbour E. Mutant BCR-ABL clones in chronic myeloid leukemia. Haematologica. 2010;96:347-9.

9. Litzow MR. Imatinib resistance: obstacles and opportunities. Arch Pathol Lab Med 2006;130:669-79.

10. Baccarani M, Cortes J, Pane F, et al. Chronic myeloid leukemia: an update of concepts and management recommendations of European LeukemiaNet. J Clin Oncol 2009;27:6041-51.

11. Gorczyca W. Cytogenetics, FISH and molecular testing in hematologic malignancies. United Kingdom: Informa Healthcare, 2008.

12. Soverini S, Martinelli G, Amabile M, et al. Denaturing-HPLC-based assay for detection of ABL mutations in chronic myeloid leukemia patients resistant to Imatinib. Clin Chem 2004;50:1205-13.

13. Shah NP, Nicoll JM, Nagar B, et al. Multiple BCR-ABL kinase domain mutations confer polyclonal resistance to the tyrosine kinase inhibitor imatinib (STI571) in chronic phase and blast crisis chronic myeloid leukemia. Cancer Cell 2002;2:11725.

14. Wongboonma W, Thongnoppakhun W,
Auewarakul CU. A single-tube allele specific-polymerase chain reaction to detect T315I resistant mutation in chronic myeloid leukemia patients. J Hematol Oncol 2011;4:7.

15. Branford S, Melo JV, Hughes TP. Selecting optimal second-line tyrosine kinase inhibitor therapy for chronic myeloid leukemia patients after imatinib failure: does the BCR-ABL mutation status really matter? Blood. 2009;114:5426-35.

16. Hughes T, Saglio G, Branford S, et al. Impact of baseline BCR-ABL mutations on response to nilotinib in patients with chronic myeloid leukemia in chronic phase. J Clin Oncol 2009;27:4204-10.

17. Quintas-Cardama A, Kantarjian H, Jones D, et al. Dasatinib (BMS-354825) is active in Philadelphia chromosome-positive chronic myelogenous leukemia after imatinib and nilotinib (AMN107) therapy failure. Blood 2007;109:497-9.

18. Ang AL, Wang ZP, Yang LY, et al.; eds. The M351T BCR-ABL Kinase Mutation Is Uncommon in Asian Patients with Imatinib-Resistant Chronic Myeloid Leukemia: Possible Relationship with Imatinib Plasma Levels. 50th ASH Annual Meeting and Exposition, San Francisco, 2008.

19. Young MA, Shah NP, Chao LH, et al. Structure of the kinase domain of an imatinib-resistant Abl mutant in complex with the Aurora kinase inhibitor VX-680. Cancer Res 2006;66:1007-14.

20. Cang S, Liu D. P-loop mutations and novel therapeutic approaches for imatinib failures in chronic myeloid leukemia. J Hematol Oncol 2008;1:15.

21. Ma W, Kantarjian H, Yeh CH, et al. BCRABL truncation due to premature translation termination as a mechanism of resistance to kinase inhibitors. Acta Haematol 2009;121:27-31.

22. Mirny LA, Gelfand MS. Using orthologous and paralogous proteins to identify specificity determining residues. Genome Biol 2002;3.

23. Ma W, Kantarjian H, Jilani I, et al. Heterogeneity in detecting Abl kinase mutations and better sensitivity using circulating plasma RNA. Leukemia 2006;20:1989-91.

24. Sherbenou DW, Hantschel 0, Kaupe I, et al. BCR-ABL SH3-SH2 domain mutations in chronic myeloid leukemia patients on imatinib. Blood 2010;116:3278-85. 